\title{
Measurement of total gamma absorption for high energy gamma rays
}

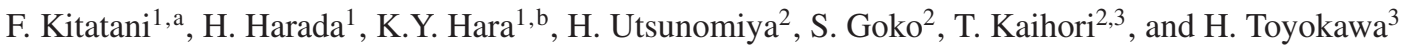 \\ 1 Japan Atomic Energy Agency, Ibaraki 319-1195, Japan \\ 2 Konan University, Kobe 658-8501, Japan \\ 3 National Institute of Advanced Industrial Science and Technology, Tsukuba 305-8551, Japan
}

\begin{abstract}
Total gamma absorption cross sections are the most important data used in various fields related to the application of gamma rays. However, the measurements of the total gamma absorption cross section were difficult for high energy gamma rays since there were no suitable gamma ray sources. Recently, the nuclear photo-absorption measurement system using the laser-Compton backscattering (LCS) gamma rays and the high-resolution high-energy photon spectrometer (HHS) were developed. We have utilized the system to measure the total gamma absorption cross section, that is, the sum of the atomic and nuclear interactions. The total gamma absorption cross sections at $10 \mathrm{MeV}$ for $\mathrm{Al}$ and $\mathrm{Cu}$ have been measured with the system. The preliminary results show that the obtained values agree fairly with the theoretical ones.
\end{abstract}

\section{Introduction}

Total gamma absorption cross sections are the most important data used in various fields related to the application of gamma rays. However, the measurements of the total gamma absorption cross section were difficult for high energy gamma rays since there were no suitable gamma ray sources. The gamma sources for such experiments in the past were

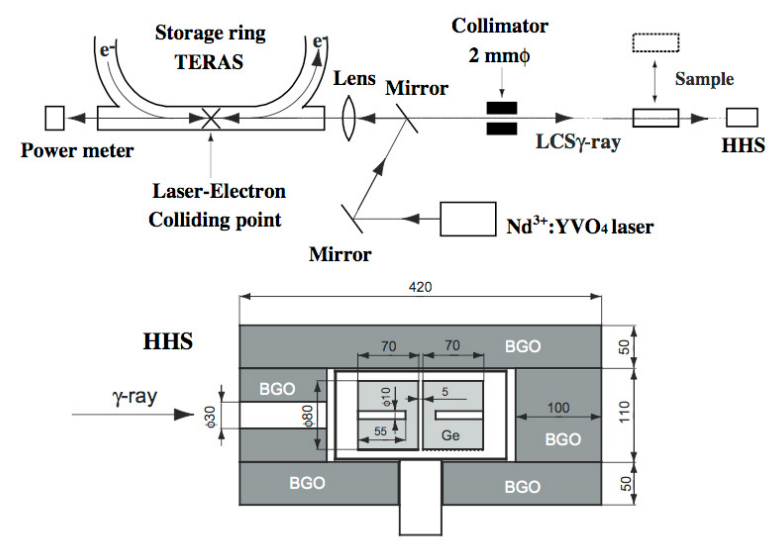

Fig. 1. Experimental setup and structure of the HHS.

neutron-capture gamma rays [1] and RI sources [2]; it was difficult to tune the energy and intensity of the gamma rays. Recently, the nuclear photo-absorption measurement system using the laser-Compton backscattering (LCS) gamma rays and the high-resolution high-energy photon spectrometer (HHS) were developed [3]. The energy and intensity of LCS gamma rays are able to be tuned, and the LCS beam be sharply collimated. Therefore, it is expected to be a powerful

\footnotetext{
${ }^{a}$ F. Kitatani, e-mail: kitatani.fumito@jaea.go.jp

${ }^{b}$ Present address: University of Tsukuba, Tsukuba 305-8577, Japan
}

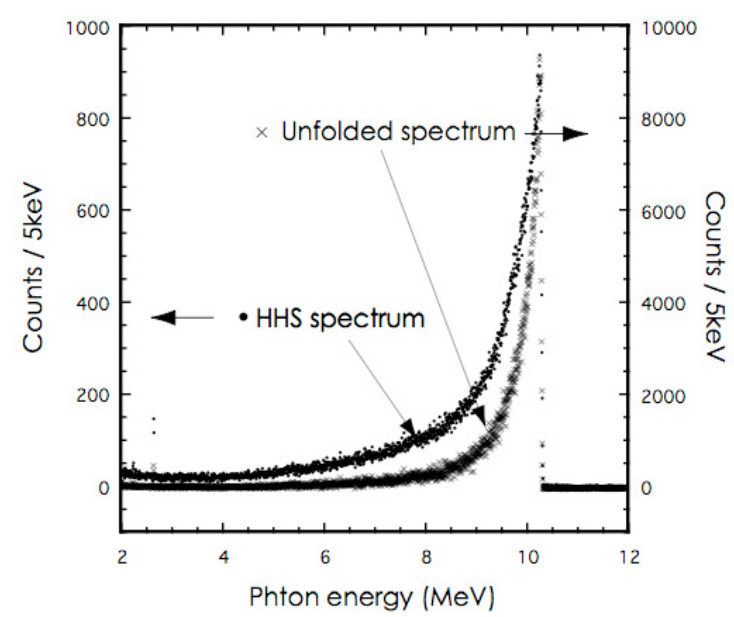

Fig. 2. LCS gamma ray spectrum (electron energy: $542.2 \mathrm{MeV}$, electron beam current: $26.0 \mathrm{~mA}$, input laser power and frequency: $24 \mathrm{~W}$ and $20 \mathrm{kHz}$ ).

tool for the measurement of the total gamma absorption cross sections. The HHS, consisting a twin Ge detector and BGO $\left(\mathrm{Bi}_{3} \mathrm{Ge}_{4} \mathrm{O}_{12}\right)$ anti-coincidence shields, made a super high-resolution spectroscopy possible. In this study, the total gamma absorption cross sections of $\mathrm{Al}$ and $\mathrm{Cu}$ at $10 \mathrm{MeV}$ were measured by using LCS gamma rays and the HHS.

\section{Experiment}

A LCS gamma ray is generated by the interaction between high energy electron and laser photon. In this study, LCS gamma rays are generated at the electron storage ring, TERAS in the National Institute of Advanced Industrial Science and Technology (AIST). The energy of electron was tuned at $542.2 \mathrm{MeV}$. The laser photon was 2 nd harmonics of $\mathrm{Nd}^{3+}: \mathrm{YVO}_{4}$ laser. The LCS gamma ray was collimated by a lead block with $2 \mathrm{~mm} \phi$ aperture. The energy distribution of 

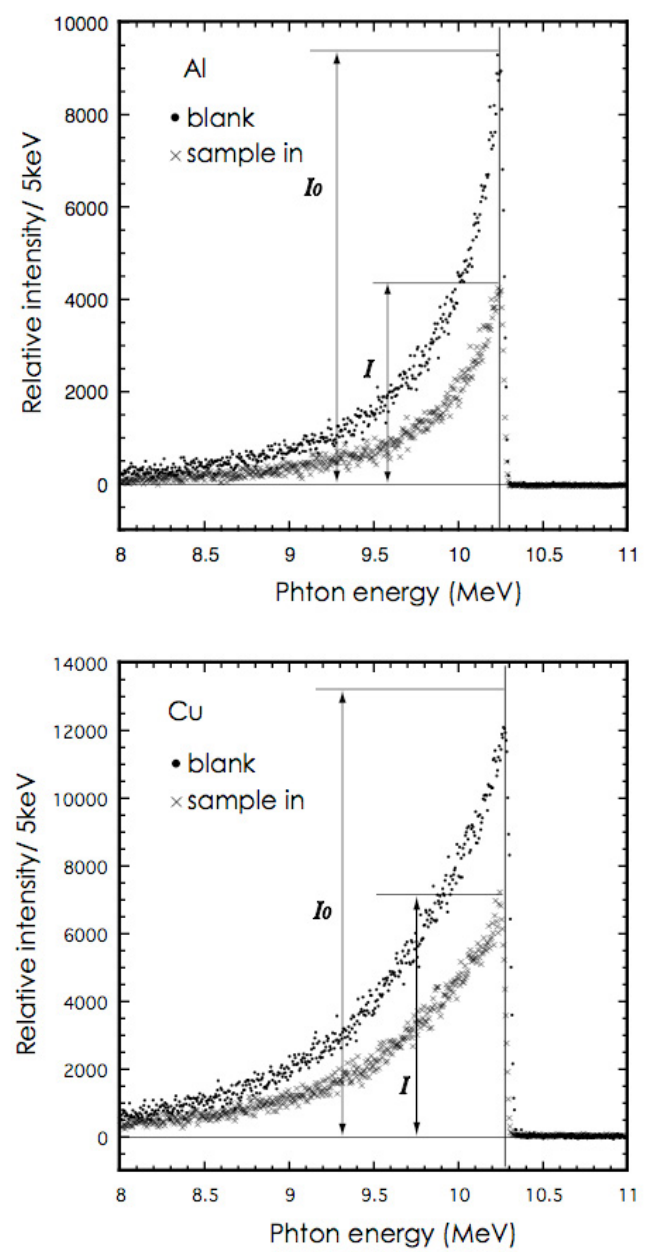

Fig. 3. Energy distributions of transmitted LCS gamma rays for the cases of an $\mathrm{Al}$ and a $\mathrm{Cu}$, sample $(\times)$ and blank $(\bullet)$.

LCS gamma rays was measured by the HHS. The HHS is a super high-resolution spectrometer consisting of a twin Ge detector and BGO anti-coincidence shields [4]. The experimental setup and the structure of the HHS are shown in figure 1 .

Total gamma absorption cross section is determined by the ratio of the intensity of the incident gamma ray to that of the attenuated gamma ray when the sample is put on the beam line. The measured samples are $\mathrm{Al}$ and $\mathrm{Cu}$. The $\mathrm{Al}$ sample is made of aluminum material A1050P specified by JIS H4000. This purity is $99.53 \%$. The size is $120 \times 20 \times 20(\mathrm{~mm})$ and the allowance is $0.1 \%$ or less. The $\mathrm{Cu}$ sample is made of copper material C1100 specified by JIS H3250. This purity is $99.96 \%$. The size is $25 \times 20 \times 20(\mathrm{~mm})$ and the allowance is $0.1 \%$ or less. To deduce the energy distribution of the LCS photon beam, the observed pulse height spectra by the HHS were unfolded by using the response function of the HHS for monochromatic photons. The response functions were calculated by using EGS4 simulation code [5]. The LCS gamma ray spectrum measured with the HHS $(\times)$ and unfolded $(\bullet)$ spectrum are shown figure 2 . The parameters of generated the LCS gamma ray were electron energy: $542.2 \mathrm{MeV}$, electron beam current: $26.0 \mathrm{~mA}$, input laser power and frequency: $24 \mathrm{~W}$ and $20 \mathrm{kHz}$.
Table 1. Calculation results of the total gamma absorption cross sections.

\begin{tabular}{|c|c|c|c|c|c|}
\hline \multicolumn{6}{|c|}{$\mathrm{Al}$ : Counts / $200 \mathrm{keV}$} \\
\hline $\mathrm{I}_{0}$ & $\operatorname{err}(\%)$ & I & $\operatorname{err}(\%)$ & $\begin{array}{l}\mu_{\text {mass }} \\
\left(\mathrm{g} / \mathrm{cm}^{2}\right)\end{array}$ & err \\
\hline 256244 & 0.33 & & & & \\
\hline 254264 & 0.23 & 121199 & 0.48 & 0.0228 & 0.0003 \\
\hline 252284 & 0.34 & 120766 & 0.35 & 0.0227 & 0.0002 \\
\hline 249650 & 0.24 & 120333 & 0.50 & 0.0225 & 0.0003 \\
\hline 247017 & 0.34 & 117394 & 0.35 & 0.0229 & 0.0002 \\
\hline 245583 & 0.24 & 114455 & 0.51 & 0.0235 & 0.0001 \\
\hline 244150 & 0.35 & & & & \\
\hline \multicolumn{6}{|c|}{$\mathrm{Al}$ : Counts / $100 \mathrm{keV}$} \\
\hline$\overline{\mathrm{I}_{0}}$ & $\operatorname{err}(\%)$ & I & $\operatorname{err}(\%)$ & $\begin{array}{l}\mu_{\text {mass }} \\
\left(\mathrm{g} / \mathrm{cm}^{2}\right)\end{array}$ & err \\
\hline 169261 & 0.88 & & & & \\
\hline 168682 & 0.62 & 79236 & 1.29 & 0.0233 & 0.0007 \\
\hline 168102 & 0.88 & 79379 & 0.91 & 0.0231 & 0.0006 \\
\hline 166802 & 0.63 & 79523 & 1.29 & 0.0228 & 0.0007 \\
\hline 165502 & 0.89 & 77429 & 0.93 & 0.0234 & 0.0006 \\
\hline 164300 & 0.63 & 75336 & 1.33 & 0.0240 & 0.0008 \\
\hline 163099 & 0.90 & & & & \\
\hline \multicolumn{6}{|c|}{$\mathrm{Cu}$ : Counts / $200 \mathrm{keV}$} \\
\hline$\overline{\mathrm{I}_{0}}$ & $\operatorname{err}(\%)$ & $\overline{\mathrm{I}}$ & $\operatorname{err}(\%)$ & $\begin{array}{l}\mu_{\text {mass }} \\
\left(\mathrm{g} / \mathrm{cm}^{2}\right)\end{array}$ & err \\
\hline 370828 & 0.58 & & & & \\
\hline 376704 & 0.41 & 194591 & 0.82 & 0.0296 & 0.0005 \\
\hline 382579 & 0.58 & 196927 & 0.58 & 0.0298 & 0.0005 \\
\hline 385042 & 0.41 & 199263 & 0.81 & 0.0295 & 0.0005 \\
\hline 387505 & 0.57 & 198623 & 0.58 & 0.0300 & 0.0005 \\
\hline 387393 & 0.41 & 197983 & 0.82 & 0.0301 & 0.0005 \\
\hline 387280 & 0.57 & & & & \\
\hline \multicolumn{6}{|c|}{$\mathrm{Cu}$ : Counts / $100 \mathrm{keV}$} \\
\hline$\overline{\mathrm{I}_{0}}$ & $\operatorname{err}(\%)$ & $\overline{\mathrm{I}}$ & $\operatorname{err}(\%)$ & $\begin{array}{l}\mu_{\text {mass }} \\
\left(\mathrm{g} / \mathrm{cm}^{2}\right)\end{array}$ & err \\
\hline 246395 & 0.70 & & & & \\
\hline 250326 & 0.49 & 130041 & 0.99 & 0.0294 & 0.0006 \\
\hline 254256 & 0.69 & 130880 & 0.70 & 0.0298 & 0.0006 \\
\hline 256344 & 0.49 & 131720 & 0.98 & 0.0299 & 0.0006 \\
\hline 258432 & 0.69 & 131754 & 0.69 & 0.0302 & 0.0006 \\
\hline 258527 & 0.49 & 131788 & 0.98 & 0.0302 & 0.0006 \\
\hline 258622 & 0.69 & & & & \\
\hline
\end{tabular}

\section{Result}

The total gamma absorption cross section, $\mu_{\text {mass }}$ is calculated by equation (1),

$$
\mu_{\text {mass }}=\frac{\ln \left(I_{0} / I\right)}{\rho \cdot T}\left[\mathrm{~g} / \mathrm{cm}^{2}\right],
$$

where $\mathrm{I}_{0}$ is incident intensity, I attenuated intensity, $\rho$ density $\left(\mathrm{g} / \mathrm{cm}^{3}\right)$, and $\mathrm{T}$ thickness $(\mathrm{cm})$. Figure 3 shows the energy distributions for the cases of an $\mathrm{Al}$ and a $\mathrm{Cu}$, sample $(\times)$ and blank $(\bullet)$.

The incident photon intensity, $\mathrm{I}_{0}$, is obtained from the HHS spectrum of a blank in beam line and the attenuated photon intensity, I, is obtained from the HHS spectrum of the sample in beam line. However, these are not able to be measured at the same time. Therefore, the measurements with a sample and without sample (blank) were repeated 

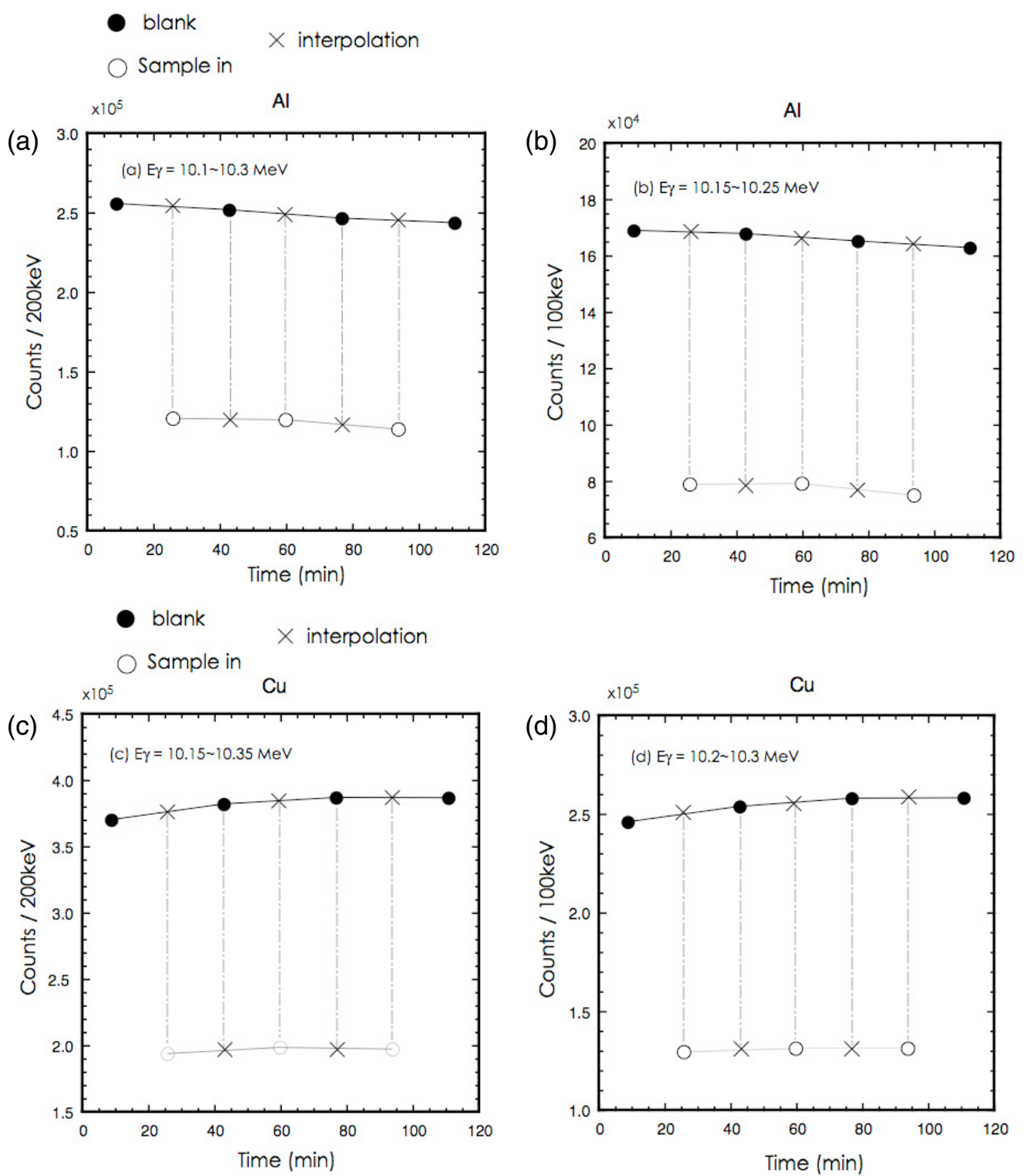

Fig. 4. Change of the measured gamma ray intensity as a function of time. (a) $\mathrm{Al}$ sample $\mathrm{E} \gamma=10.1 \sim 10.3 \mathrm{MeV}$, (b) $\mathrm{Al}$ sample $\mathrm{E} \gamma=10.15 \sim$ 10.2.5 MeV, (c) Cu sample $\mathrm{E} \gamma=10.15 \sim 10.35 \mathrm{MeV}$, (d) $\mathrm{Cu}$ sample $\mathrm{E} \gamma=10.2 \sim 10.3 \mathrm{MeV}$.

Table 2. Time average of the result at each measurements.

\begin{tabular}{lllll}
\hline $\mathrm{Al}:$ & $10.2 \mathrm{keV}$ & $\mathrm{MeV} / 200$ & $\mu_{\text {mass }}:$ & $0.0229 \pm 0.0004\left(\mathrm{~g} / \mathrm{cm}^{2}\right)$ \\
$\mathrm{Al}:$ & $10.2 \mathrm{keV}$ & $\mathrm{MeV} / 100$ & $\mu_{\text {mass }}:$ & $0.0233 \pm 0.0004\left(\mathrm{~g} / \mathrm{cm}^{2}\right)$ \\
\hline $\mathrm{Cu}:$ & $10.25 \mathrm{keV}$ & $\mathrm{MeV} / 200$ & $\mu_{\text {mass }}:$ & $0.0298 \pm 0.0002\left(\mathrm{~g} / \mathrm{cm}^{2}\right)$ \\
$\mathrm{Cu}:$ & $10.25 \mathrm{keV}$ & $\mathrm{MeV} / 100$ & $\mu_{\text {mass }}:$ & $0.0299 \pm 0.0004\left(\mathrm{~g} / \mathrm{cm}^{2}\right)$ \\
\hline
\end{tabular}

alternately during the same measurement period. $\mathrm{I}_{0}$ and $\mathrm{I}$ at same time were determined by interpolation.

The change of the measured gamma ray intensity as a function of time is shown in figure $4(\mathrm{a}-\mathrm{d})$. The results are shown for each sample in both case of the width of energy $100 \mathrm{keV}$ and $200 \mathrm{keV}$. In all the measurements, the measurement time is $15 \mathrm{~min}$ and the interval time is $2 \mathrm{~min}$.

The calculated results of the total gamma absorption cross sections are shown in table 1 .

The results on $\mathrm{Al}$ agree with the theoretical value $\left(0.0232 \mathrm{~g} / \mathrm{cm}^{2}\right)$ [6] within the limits of error. The results on
$\mathrm{Cu}$ are about 3\% smaller than the theoretical value $\left(0.0310 \mathrm{~g} / \mathrm{cm}^{2}\right)$ [6]. The detailed examination on systematic errors will be done in near future.

\section{Conclusion}

Total gamma absorption cross sections, the most important data used in various fields related to the application of gamma rays, nuclear data, radiation shield, etc., were measured by the method using LCS gamma rays and the HHS of Al and $\mathrm{Cu}$ at the vicinity of $10 \mathrm{MeV}$. Results for both samples agree well with the theoretical values. The effectiveness of the measurement method using the LCS gamma rays and the HHS were demonstrated for the total gamma absorption cross sections. It is scheduled that detailed examination of the systematic error analysis will be done, and a systematic 
measurement will be executed on the total gamma absorption cross sections in the future.

Next, the total gamma absorption cross sections obtained by taking the average of the results at each measurements are shown in table 2 . The errors include only statistical ones.

\section{References}

1. R.H. Barlett, D.J. Donahue, Phys. Rev. 137, A532 (1965).

2. T.K. Umesh, C. Ranganathaiah, R. Gowda, K.S. Puttaswamy, B. Sanjeevaiah, Phys. Rev. A 23, 2365 (1981).
3. H. Harada, Y.S higetome, J. Nucl. Sci. Technol. 32, 1189 (1995).

4. H. Harada, K. Furutaka, S. Nakamura, K. Osaka, H. Akimune, H. Utsunomiya, T. Ohsaki, M. Igashira, Nucl. Instrum. Meth. A 554, 306 (2005).

5. F. Kitatani, H. Harada, K.Y. Hara, M. Ohsima, H. Utsunomiya, K. Yamada, H. Toyokawa, in Proceedings of 2006 Fall Meeting of the Atomic Energy Society of Japan, p. 214.

6. M.J. Berger, J.H. Hubbell, S.M. Seltzer, J. Chang, S. Coursey, R. Sukumar, D.S. Zucker Smith, XCOM: Photon Cross Sections Database, NIST.

(http://www.physics.nist.gov/PhysRefData/Xcom/Text/XCOM. html). 